\title{
Counting intersecting and pairs of cross-intersecting families
}

\author{
Peter Frankl, Andrey Kupavskii*
}

\begin{abstract}
A family of subsets of $\{1, \ldots, n\}$ is called intersecting if any two of its sets intersect. A classical result in extremal combinatorics due to Erdős, Ko, and Rado determines the maximum size of an intersecting family of $k$-subsets of $\{1, \ldots, n\}$. In this paper we study the following problem: how many intersecting families of $k$-subsets of $\{1, \ldots, n\}$ are there? Improving a result of Balogh, Das, Delcourt, Liu, and Sharifzadeh, we determine this quantity asymptotically for $n \geq 2 k+2+2 \sqrt{k \log k}$ and $k \rightarrow \infty$. Moreover, under the same assumptions we also determine asymptotically the number of non-trivial intersecting families, that is, intersecting families for which the intersection of all sets is empty. We obtain analogous results for pairs of cross-intersecting families.
\end{abstract}

MSc classification: 05D05

\section{Introduction}

A family is a collection of subsets of an $n$-element set $[n]$. Collections $\mathcal{F} \subset\left(\begin{array}{c}{[n]} \\ k\end{array}\right)$ are called $k$-uniform families. A family $\mathcal{F}$ is called intersecting if $F \cap F^{\prime} \neq \emptyset$ holds for all $F, F^{\prime} \in \mathcal{F}$. Similarly, $\mathcal{F} \subset\left(\begin{array}{c}{[n]} \\ k\end{array}\right)$ and $\mathcal{G} \subset\left(\begin{array}{c}{[n]} \\ l\end{array}\right)$ are called cross-intersecting, if for all $F \in \mathcal{F}, G \in \mathcal{G}$ one has $F \cap G \neq \emptyset$.

The research concerning intersecting families was initiated by Erdős, Ko and Rado, who determined the maximum size of intersecting families.

Theorem 1 (Erdős, Ko, Rado [3]). Suppose that $n \geq 2 k>0$ and $\mathcal{F} \subset\left(\begin{array}{c}{[n]} \\ k\end{array}\right)$ is intersecting. Then

$$
|\mathcal{F}| \leq\left(\begin{array}{l}
n-1 \\
k-1
\end{array}\right)
$$

The family of all $k$-sets containing a fixed element shows that (1) is best possible. Hilton and Milner proved in a stronger form that for $n>2 k$ these are the only families on which the equality is attained. We say that an intersecting family $\mathcal{F}$ is non-trivial if $\bigcap_{F \in \mathcal{F}} F=\emptyset$, that is, if it cannot be pierced by a single point.

${ }^{*}$ Moscow Institute of Physics and Technology, Ecole Polytechnique Fédérale de Lausanne; Email: kupavskii@yandex.ru Research of Andrey Kupavskii is supported by the grant RNF 16-11-10014. 
Theorem 2 (Hilton, Milner [7]). Let $n>2 k>0$ and suppose that $\mathcal{F} \subset\left(\begin{array}{c}{[n]} \\ k\end{array}\right)$ is a non-trivial intersecting family. Then

$$
|\mathcal{F}| \leq\left(\begin{array}{c}
n-1 \\
k-1
\end{array}\right)-\left(\begin{array}{c}
n-k-1 \\
k-1
\end{array}\right)+1
$$

For $n=2 k+1$ the difference between the upper bounds (11) and (2) is only $k-1$. However, as $n-2 k$ increases, this difference gets much larger. The number of subfamilies of $\mathcal{F}$ is $2^{|\mathcal{F}|}$, and thus the ratio between the number of subfamilies of the Erdös-Ko-Rado family and that of the Hilton-Milner family is $2^{k-1}$ for $n=2 k+1$ and grows very fast as $n-2 k$ increases. This serves as an indication that most intersecting families are trivial, i.e., satisfy $\bigcap_{F \in \mathcal{F}} F \neq \emptyset$.

In an important recent paper Balogh, Das, Delcourt, Liu, and Sharifzadeh [1] proved this in the following quantitative form. Let $I(n, k)$ denote the total number of intersecting families $\mathcal{F} \subset\left(\begin{array}{c}{[n]} \\ k\end{array}\right)$.

Theorem 3 (Balogh, Das, Delcourt, Liu, and Sharifzadeh [1]). If $n \geq 3 k+8 \log k$ then

$$
I(n, k)=(n+o(1)) 2^{\left(\begin{array}{c}
n-1 \\
k-1
\end{array}\right)},
$$

where $o(1) \rightarrow 0$ as $k \rightarrow \infty$.

One of the main tools of the proof of (3) is a nice bound on the number of maximal (i.e., non-extendable) intersecting families (see Lemma 10). They obtain this bound using the following fundamental result of Bollobás.

Theorem 4 (Bollobás [2]). Suppose that $\mathcal{A} \subset\left(\begin{array}{c}{[n]} \\ a\end{array}\right), \mathcal{B} \subset\left(\begin{array}{c}{[n]} \\ b\end{array}\right)$ with $\mathcal{A}=\left\{A_{1}, \ldots, A_{m}\right\}, \mathcal{B}=$ $\left\{B_{1}, \ldots, B_{m}\right\}$ satisfy $A_{i} \cap B_{j}=\emptyset$ iff $i=j$. Then

$$
m \leq\left(\begin{array}{c}
a+b \\
a
\end{array}\right) .
$$

Note that the bound (4) is independent of $n$. In [2] it is proved in a more general setting, not requiring uniformity, i.e., for $\mathcal{A}, \mathcal{B} \subset 2^{[n]}$. The uniform version (4) was rediscovered several years later by Jaeger-Payan [8] and Katona [10].

We are going to use (4) to obtain an upper bound on the number of maximal pairs of cross-intersecting families. Let us denote by $C I(n, a, b, t)\left(C I\left(n, a, b,\left[t_{1}, t_{2}\right]\right)\right)$ the number of pairs of cross-intersecting families $\mathcal{A} \subset\left(\begin{array}{c}{[n]} \\ a\end{array}\right), \mathcal{B} \subset\left(\begin{array}{c}{[n]} \\ b\end{array}\right)$ with $|\mathcal{A}|=t\left(t_{1} \leq|\mathcal{A}| \leq t_{2}\right)$. We also denote $C I(n, a, b):=\sum_{t} C I(n, a, b, t)$.

We prove the following bound for the number of pairs of cross-intersecting families.

Theorem 5. Choose $a, b, n \in \mathbb{N}$ and put $c:=\max \{a, b\}, T:=\left(\begin{array}{c}n-a+b-1 \\ n-a\end{array}\right)$. For $n \geq a+b+$ $2 \sqrt{c \log c}+2 \max \{0, a-b\}, a, b \rightarrow \infty$, and $b \gg \log a$ we have

$$
\begin{aligned}
C I(n, a, b) & =\left(1+\delta_{a b}+o(1)\right) 2^{\left(\begin{array}{l}
n \\
c
\end{array}\right),} \\
C I(n, a, b,[1, T]) & =(1+o(1))\left(\begin{array}{l}
n \\
a
\end{array}\right) 2^{\left(\begin{array}{c}
n \\
b
\end{array}\right)-\left(\begin{array}{c}
n-a \\
b
\end{array}\right),}
\end{aligned}
$$

where $\delta_{a b}=1$ if $a=b$, and 0 otherwise. 
In fact, (6) easily implies (5)) (see Section 2 for details).

For a family $\mathcal{F} \subset\left(\begin{array}{c}{[n]} \\ k\end{array}\right)$ we define the diversity $\gamma(\mathcal{F})$ of $\mathcal{F}$ to be $|\mathcal{F}|-\Delta(\mathcal{F})$, where $\Delta(\mathcal{F}):=\max _{i \in[n]}|\{F: i \in F \in \mathcal{F}\}|$. For an integer $t$ denote by $I(n, k, t)(I(n, k, \geq t))$ the number of intersecting families with diversity $t$ (at least $t$ ). In particular, $I(n, k, \geq 1$ ) is the number of non-trivial intersecting families. With the help of (6) we obtain a refinement of Theorem 3 .

Theorem 6. For $n \geq 2 k+2+2 \sqrt{k \log k}$ and $k \rightarrow \infty$ we have

$$
\begin{aligned}
I(n, k) & =(n+o(1)) 2^{\left(\begin{array}{c}
n-1 \\
k-1
\end{array}\right)}, \\
I(n, k, \geq 1) & =(1+o(1)) n\left(\begin{array}{c}
n-1 \\
k
\end{array}\right) 2^{\left(\begin{array}{c}
n-1 \\
k-1
\end{array}\right)-\left(\begin{array}{c}
n-k-1 \\
k-1
\end{array}\right) .}
\end{aligned}
$$

Again, it is easy to see that (8) implies (7) (see Section 3 for details). In the next section we present the proof of Theorem 5, and in Section 3 we give the proof of Theorem [6,

\section{Cross-intersecting families}

Let us define the lexicographic order on the $k$-subsets of $[n]$. We have $F \prec G$ in the lexicographic order if $\min F \backslash G<\min G \backslash F$ holds. E.g., $\{1,10\} \prec\{2,3\}$. For $0 \leq m \leq\left(\begin{array}{l}n \\ k\end{array}\right)$ let $\mathcal{L}^{(k)}(m)$ denote the family of first $m k$-sets in the lexicographic order. E.g., $\mathcal{L}^{(k)}\left(\left(\begin{array}{c}n-1 \\ k-1\end{array}\right)\right)=$ $\left\{F \in\left(\begin{array}{c}{[n]} \\ k\end{array}\right): 1 \in F\right\}$.

Next we state the Kruskal-Katona Theorem [11], [9], which is one of the most important results in extremal set theory.

Theorem 7 (Kruskal [11], Katona [9]). If $\mathcal{A} \subset\left(\begin{array}{c}{[n]} \\ a\end{array}\right)$ and $\mathcal{B} \subset\left(\begin{array}{c}{[n]} \\ b\end{array}\right)$ are cross-intersecting then $\mathcal{L}^{(a)}(|\mathcal{A}|)$ and $\mathcal{L}^{(b)}(|\mathcal{B}|)$ are cross-intersecting as well.

Computationwise, the bounds arising from the Kruskal-Katona Theorem are not easy to handle. Lovász [13] found a slightly weaker but very handy form, which may be stated as follows.

Theorem 8 (Lovász [13]). Let $n \geq a+b$, and consider a pair of cross-intersecting families

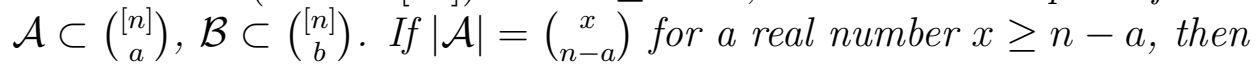

$$
|\mathcal{B}| \leq\left(\begin{array}{l}
n \\
b
\end{array}\right)-\left(\begin{array}{l}
x \\
b
\end{array}\right) \quad \text { holds }
$$

Note that for $x \geq k-1$ the polynomial $\left(\begin{array}{l}x \\ k\end{array}\right)$ is a monotone increasing function of $x$. Thus $x$ is uniquely determined by $|\mathcal{A}|$ and $a$.

We would also need the following result, which proof is based on Theorem 7 and which is a combination of a result of Frankl and Tokushige [5] (Theorem 2 in [5]) and two results of Kupavskii and Zaharov [12] (Part 1 of Theorem 1 and Corollary 1). 
Theorem 9 (Frankl, Tokushige, [5, Kupavskii, Zakharov [12]). Let $n>a+b$ and suppose that the families $\mathcal{A} \subset\left(\begin{array}{c}{[n]} \\ a\end{array}\right), \mathcal{B} \subset\left(\begin{array}{c}{[n]} \\ b\end{array}\right)$ are cross-intersecting. If for some real number $\alpha \geq 1$ we have $\left(\begin{array}{c}n-\alpha \\ n-a\end{array}\right) \leq|\mathcal{A}| \leq\left(\begin{array}{c}n-a+b-1 \\ n-a\end{array}\right)$, then

$$
|\mathcal{A}|+|\mathcal{B}| \leq\left(\begin{array}{l}
n \\
b
\end{array}\right)+\left(\begin{array}{l}
n-\alpha \\
a-\alpha
\end{array}\right)-\left(\begin{array}{c}
n-\alpha \\
b
\end{array}\right) .
$$

Note that the upper bound on $|\mathcal{A}|$ in this theorem is exactly the same as in (6).

We go on to the proof of Theorem 5. First we show that (6) implies (5). We may w.l.o.g. assume for this paragraph that $c=b \geq a$. For $b>a$ we have $T \geq\left(\begin{array}{l}n \\ a\end{array}\right)$ and thus $C I(n, a, b, t)=0$ for $t>T$. Therefore, we have $C I(n, a, b)=C I(n, a, b, 0)+C I(n, a, b,[1, T])$. If $a=b$, then $T=\left(\begin{array}{c}n-1 \\ a-1\end{array}\right)$, and it follows from Theorem 7 that if $\mathcal{A}, \mathcal{B} \subset\left(\begin{array}{c}{[n]} \\ a\end{array}\right)$ are crossintersecting, then $\min \{|\mathcal{A}|,|\mathcal{B}|\} \leq\left(\begin{array}{c}n-1 \\ a-1\end{array}\right)$. Therefore, in the case $a=b$ we have

$$
2 C I(n, a, b, 0)-1 \leq C I(n, a, b) \leq 2(C I(n, a, b, 0)+C I(n, a, b,[1, T])) .
$$

The " -1 " in the first inequality stands for a pair of empty families, which is counted twice.

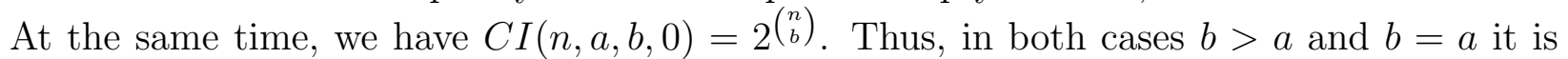
sufficient to show that the right-hand side of (6) $)$ is $o\left(2^{\left(\begin{array}{l}n \\ b\end{array}\right)}\right)$. We first note that $n-a-b \geq \sqrt{n}$ for $b \geq a, n \geq a+b+2 \sqrt{b \log b}$, since $4 b \log b \geq a+b+2 \sqrt{b \log b}$. The rest is done by a simple calculation:

$$
\frac{C I(n, a, b,[1, T])}{2^{\left(\begin{array}{c}
n \\
b
\end{array}\right)}}=\left(\begin{array}{c}
n \\
a
\end{array}\right) 2^{-\left(\begin{array}{c}
n-a \\
b
\end{array}\right)} \leq 2^{n-\left(\begin{array}{c}
b+\sqrt{n} \\
b
\end{array}\right)}=o(1) .
$$

Next, discuss the proof of the lower bound in (66). To obtain that many pairs of intersecting families, take $\mathcal{A}:=\{A\}, A \in\left(\begin{array}{c}{[n]} \\ a\end{array}\right)$ and $\mathcal{B}(A):=\left\{B \in\left(\begin{array}{c}{[n]} \\ b\end{array}\right): B \cap A \neq \emptyset\right\}$. Next, choose an arbitrary subfamily $\mathcal{B} \subset \mathcal{B}(A)$. We only need to assure that few of these pairs of subfamilies are counted twice. Actually, we count a pair of families twice only in the case when $a=b$ and both $\mathcal{A}, \mathcal{B}$ consist of one set. The number of such pairs is $\left(\begin{array}{c}{[n]} \\ a\end{array}\right)^{2}$ and is negligible compared to the right hand side of (6) $)$.

We pass to the proof of the upper bound.

$\mathbf{2} \leq|\mathcal{A}| \leq \boldsymbol{n}-\boldsymbol{a}$. Applying Theorem $\mathbf{7}$, the size of the (unique) maximal family $\mathcal{B}^{\prime}$ that forms a cross-intersecting pair with $\mathcal{A}$ is maximized if $\mathcal{A}$ consists of two sets $A_{1}, A_{2}$ that intersect in $a-1$ elements. Therefore, $\left|\mathcal{B}^{\prime}\right| \leq\left(\begin{array}{c}n \\ b\end{array}\right)-\left(\begin{array}{c}n-a+1 \\ b\end{array}\right)+\left(\begin{array}{c}n-a-1 \\ b-2\end{array}\right)$. Any other family $\mathcal{B}$ that forms a cross-intersecting pair with $\mathcal{A}$ must be a subfamily of $\mathcal{B}^{\prime}$.

So we can bound the number of pairs of cross-intersecting families $\mathcal{A}, \mathcal{B}$ with $2 \leq|\mathcal{A}| \leq$ $n-a$ as follows:

$$
\frac{\sum_{t=2}^{n-a} C I(n, a, b, t)}{2^{\left(\begin{array}{c}
n \\
b
\end{array}\right)-\left(\begin{array}{c}
n-a \\
b
\end{array}\right)}} \leq \sum_{t=2}^{n-a}\left(\begin{array}{c}
n \\
a \\
t
\end{array}\right) \frac{2^{\left(\begin{array}{c}
n \\
b
\end{array}\right)-\left(\begin{array}{c}
n-a+1 \\
b
\end{array}\right)+\left(\begin{array}{c}
n-a-1 \\
b-2
\end{array}\right)}}{2^{\left(\begin{array}{c}
n \\
b
\end{array}\right)-\left(\begin{array}{c}
n-a \\
b
\end{array}\right)}} \leq 2^{n^{2}} 2^{-\left(\begin{array}{c}
n-a-1 \\
b-1
\end{array}\right)}=o(1) .
$$


$\boldsymbol{n}-\boldsymbol{a}+\mathbf{1} \leq|\mathcal{A}| \leq\left(\begin{array}{c}\boldsymbol{n}-\boldsymbol{u} \\ \boldsymbol{n}-\boldsymbol{a}\end{array}\right)$, where $u=\sqrt{c \log c}+\max \{0, a-b\}$. Note that $n-a+1=$ $\left(\begin{array}{c}n-a+1 \\ n-a\end{array}\right)$. In this case the bound is similar, but we use Theorem 8 to bound the size of $|\mathcal{B}|$.

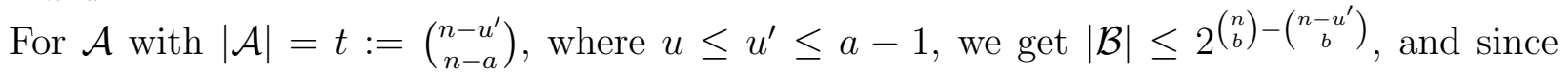
$\left(\begin{array}{c}n \\ a \\ t\end{array}\right) \leq 2^{n} t$, we have the following bound:

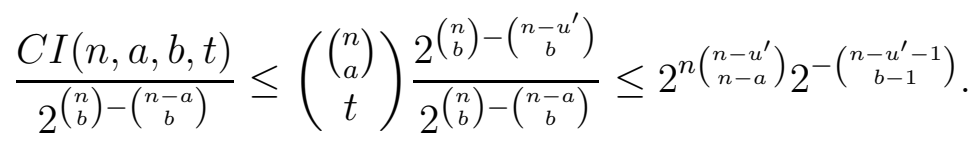

At the same time we have $n \geq a+b+2 u$ and

$$
\begin{aligned}
\frac{\left(\begin{array}{c}
n-u^{\prime} \\
n-a
\end{array}\right)}{\left(\begin{array}{c}
n-u^{\prime}-1 \\
b-1
\end{array}\right)}=\frac{n-u^{\prime}}{b} \prod_{i=0}^{n-a-b-1} \frac{n-b-u^{\prime}-i}{n-a-i} \leq \\
\frac{n}{b} \prod_{i=0}^{n-a-b-1} \frac{n-a-\sqrt{c \log c}-i}{n-a-i} \leq \frac{n}{b} e^{-\sqrt{c \log c}\left(\sum_{i=b+1}^{n-a} \frac{1}{i}\right)} \leq \frac{1}{2 n}
\end{aligned}
$$

for sufficiently large $c$. Indeed, $\sqrt{c \log c} \sum_{i=b+1}^{n-a} \frac{1}{i} \geq \sqrt{c \log c} \sum_{i=b+1}^{b+2 \sqrt{c \log c}} \frac{1}{i} \geq(1+o(1)) \frac{2(\sqrt{c \log c})^{2}}{c}=$ $(2+o(1)) \log c$, which justifies (11) for $n \leq b^{3 / 2}$. For $n>b^{3 / 2}$ we have $\sqrt{c \log c} \sum_{i=b+1}^{n-a} \frac{1}{i} \geq$ $(1+o(1)) \sqrt{c \log c} \log \frac{n}{b} \geq(1+o(1)) \sqrt{c \log c} \log n^{2 / 3} \gg \log n$, which justifies (11) for $n>b^{3 / 2}$.

We conclude that

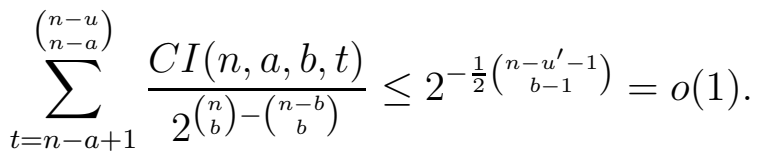

$\left(\begin{array}{l}\boldsymbol{n}-\boldsymbol{u} \\ \boldsymbol{n}-\boldsymbol{a}\end{array}\right)<|\mathcal{A}| \leq T$, where $u=\sqrt{b \log b}+\max \{0, a-b\}$. Using the Bollobas set-pair inequality, it is not difficult to obtain the following bound on the number of maximal pairs of cross-intersecting families.

Lemma 10. The number of maximal cross-intersecting pairs $\mathcal{A}^{\prime} \subset\left(\begin{array}{c}{[n]} \\ a\end{array}\right), \mathcal{B}^{\prime} \subset\left(\begin{array}{c}{[n]} \\ b\end{array}\right)$ is at most $\left[\left(\begin{array}{l}n \\ a\end{array}\right)\left(\begin{array}{l}n \\ b\end{array}\right)\right]^{\left(\begin{array}{c}a+b \\ a\end{array}\right)}$.

We note that the proof is very similar to the proof of an analogous statement for intersecting families from [1].

Proof. Find a minimal $\mathcal{B}^{\prime}$-generating family $\mathcal{M} \subset \mathcal{A}^{\prime}$ such that $\mathcal{B}^{\prime}=\left\{B \in\left(\begin{array}{c}{[n]} \\ b\end{array}\right): B \cap M \neq\right.$ $\emptyset$ for all $M \in \mathcal{M}\}$. We claim that $|\mathcal{M}| \leq\left(\begin{array}{c}a+b \\ a\end{array}\right)$. Indeed, due to minimality, for each set $M^{\prime} \in \mathcal{M}$ the family $\mathcal{B}^{\prime \prime}:=\left\{B \in\left(\begin{array}{c}{[n]} \\ b\end{array}\right): B \cap M \neq \emptyset\right.$ for all $\left.M \in \mathcal{M}-\left\{M^{\prime}\right\}\right\}$ strictly contains $\mathcal{B}^{\prime}$. Therefore, there is a set $B$ in $\mathcal{B}^{\prime \prime} \backslash \mathcal{B}^{\prime}$ such that $B \cap M^{\prime}=\emptyset, B \cap M \neq \emptyset$ for all $M \in \mathcal{M}-\left\{M^{\prime}\right\}$. Applying the inequality (4) to $\mathcal{M}$ and the collection of such sets $B$, we get that $|\mathcal{M}| \leq\left(\begin{array}{c}a+b \\ b\end{array}\right)$. 
Interchanging the roles of $\mathcal{A}^{\prime}$ and $\mathcal{B}^{\prime}$, we get that a minimal $\mathcal{A}^{\prime}$-generating family has size at most $\left(\begin{array}{c}a+b \\ b\end{array}\right)$ as well. Now the bound stated in the lemma is just a crude upper bound on the number of ways one can choose these two generating families out of $\left(\begin{array}{c}{[n]} \\ a\end{array}\right)$ and $\left(\begin{array}{c}{[n]} \\ b\end{array}\right)$, respectively.

Combined with the bound (10) on the size of any maximal pair of families with such cardinalities, we get that

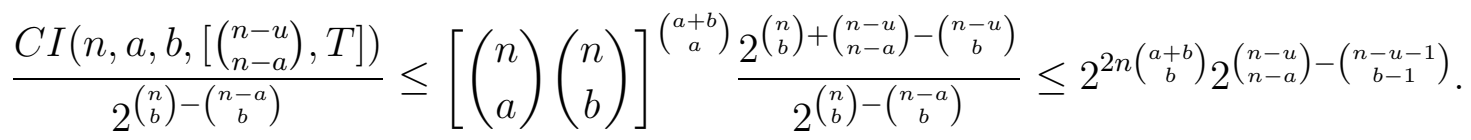

We also have

$$
\frac{\left(\begin{array}{c}
a+b \\
b
\end{array}\right)}{\left(\begin{array}{c}
n-u-1 \\
b-1
\end{array}\right)}=\frac{n-u}{b} \prod_{i=0}^{b-1} \frac{a+b-i}{n-u-i} \leq n\left(\frac{a+b}{n-u}\right)^{b} \leq \frac{1}{4 n}
$$

Indeed, the last inequality is clearly valid for $n \geq(a+b)^{2}, b \rightarrow \infty$. If $n<(a+b)^{2}$, then the before-last expression is at most

$$
e^{\log n-\frac{b(n-a-b-u)}{n-u}} \leq e^{2 \log (a+b)-\frac{b(u+\max \{0, a-b\})}{O(a+b)}} \leq e^{2 \log (a+b)-\Omega(\min \{b, u\})} .
$$

Since by the assumption we have $b \gg \log (a+b)$ and also, obviously, $u \gg \log (a+b)$, the last expression is at most $e^{-4 \log (a+b)}<\frac{1}{4 n}$.

Taking into account (11), which is valid for $u^{\prime}=u$, we conclude that the right-hand side of (12) is $o(1)$.

\section{$3 \quad$ Intersecting families}

We need a theorem due to Frankl [4], proved in the following, slightly stronger, form in [12].

Theorem 11 ([4, 12] ). Let $\mathcal{F} \subset\left(\begin{array}{c}{[n]} \\ k\end{array}\right)$ be an intersecting family, and $n>2 k$. Then, if $\gamma(\mathcal{F}) \geq\left(\begin{array}{c}n-u-1 \\ k-u\end{array}\right)$ for some real $3 \leq u \leq k$, then

$$
|\mathcal{F}| \leq\left(\begin{array}{c}
n-1 \\
k-1
\end{array}\right)+\left(\begin{array}{c}
n-u-1 \\
k-u
\end{array}\right)-\left(\begin{array}{c}
n-u-1 \\
k-1
\end{array}\right)
$$

We go on to the proof Theorem 6. Let us first show that (8) implies (7). Indeed, using that $n-2 k-1 \geq \sqrt{n}$ and $k \rightarrow \infty$ in the assumptions of Theorem 6 , we get

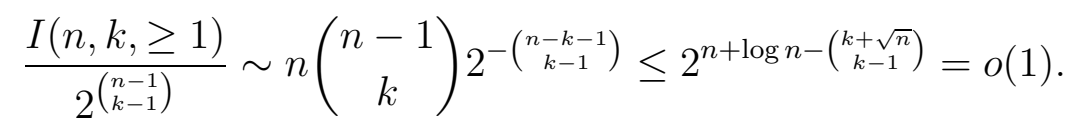


Therefore, $I(n, k, \geq 1)=o\left(2^{\left(\begin{array}{c}n-1 \\ k-1\end{array}\right)}\right)$. On the other hand, it is easy to see that $I(n, k, 0)=$ $(n+o(1)) 2^{\left(\begin{array}{l}n-1 \\ k-1\end{array}\right)}$ (for the proof see [1]).

Let us prove the lower bound in (8) $)$. For $S \in\left(\begin{array}{c}{[n]} \\ k\end{array}\right), i \in[n] \backslash S$ define the family $\mathcal{H}(i, S):=$ $\{S\} \cup\left\{H \in\left(\begin{array}{c}{[n]} \\ k\end{array}\right): i \in H, H \cap S \neq \emptyset\right\}$. Due to Theorem 2, these families are the largest non-trivial intersecting families. We have $|\mathcal{H}(i, s)|=\left(\begin{array}{c}n-1 \\ k-1\end{array}\right)-\left(\begin{array}{c}n-k-1 \\ k-1\end{array}\right)+1$, and each such family contains no less than

$$
2^{\left(\begin{array}{c}
n-1 \\
k-1
\end{array}\right)-\left(\begin{array}{c}
n-k-1 \\
k-1
\end{array}\right)}-k 2^{\left(\begin{array}{c}
n-2 \\
k-2
\end{array}\right)}=(1+o(1)) 2^{\left(\begin{array}{c}
n-1 \\
k-1
\end{array}\right)-\left(\begin{array}{c}
n-k-1 \\
k-1
\end{array}\right)}
$$

non-trivial intersecting subfamilies, as $k \rightarrow \infty$. Indeed, a subfamily of $\mathcal{H}(i, S)$ containing $S$ is non-trivial unless all sets containing $i$ contain also a fixed $j \in S$. In other words, they must be a subset of a family $\mathcal{I}(i, j, S):=\{S\} \cup\left\{I \in\left(\begin{array}{c}{[n]} \\ k\end{array}\right): i, j \in S\right\}$. The number of subfamilies

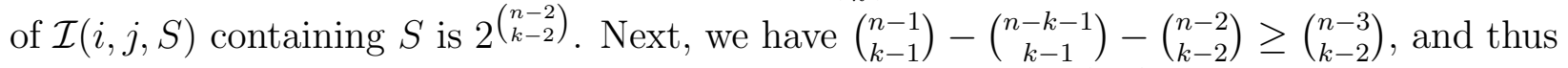
the last inequality in the displayed formula above holds since $2^{\left(\begin{array}{l}n-3 \\ k-2\end{array}\right)} \gg k$. Denote the set of all non-trivial subfamilies of $\mathcal{H}(i, S)$ by $\tilde{\mathcal{H}}(i, S)$.

Therefore, $\sum_{S \in\left(\begin{array}{c}n \\ k\end{array}\right), i \notin S}|\tilde{\mathcal{H}}(i, S)|=(1+o(1)) n\left(\begin{array}{c}n-1 \\ k\end{array}\right) 2\left(\begin{array}{c}n-1 \\ k-1\end{array}\right)-\left(\begin{array}{c}n-k-1 \\ k-1\end{array}\right)$. On the other hand, the pairwise intersections of these families are small: the families from $\tilde{\mathcal{H}}(i, S) \cap \tilde{\mathcal{H}}\left(i, S^{\prime}\right)$ form the set $I(n, k, 2)$, and we do (somewhat implicitly) show in the proof that $I(n, k, 2)=$ $o(I(n, k, 1))$. It could also be verified by a simple direct, but somewhat tedious calculation. Therefore, the lower bound is justified.

Next we prove the upper bound. For $i \in[n]$ and $\mathcal{F} \subset\left(\begin{array}{c}{[n]} \\ k\end{array}\right)$ we use the standard notation

$$
\begin{aligned}
& \mathcal{F}(i):=\{F-\{i\}: i \in F \in \mathcal{F}\} \subset\left(\begin{array}{c}
{[n]-\{i\}} \\
k-1
\end{array}\right), \\
& \mathcal{F}(\bar{i}):=\{F \in \mathcal{F}: i \notin F\} \subset\left(\begin{array}{c}
{[n]-\{i\}} \\
k
\end{array}\right) .
\end{aligned}
$$

Note that if $\mathcal{F}$ is intersecting then $\mathcal{F}(i)$ and $\mathcal{F}(\bar{i})$ are cross-intersecting.

We count the number of families with different diversity separately. The number of families $\mathcal{F}$ with $i$ being the most popular element and $\gamma(\mathcal{F}) \leq\left(\begin{array}{l}n-3 \\ k-2\end{array}\right)$ is at most the number of cross-intersecting pairs $\mathcal{F}(\bar{i}), \mathcal{F}(i)$.

Therefore, we may apply (6) with $n^{\prime}:=n-1, a:=k, b:=k-1$, and get that the number

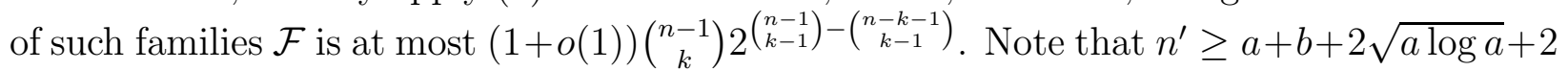
and, in terms of Theorem 5, we have $T=\left(\begin{array}{l}n-3 \\ k-2\end{array}\right)$ for our case. Multiplying the number of such families by the number of choices of $i$, we get the claimed asymptotic.

We are only left to prove that there are few families with diversity larger than $\left(\begin{array}{l}n-3 \\ k-2\end{array}\right)$. Using the upper bound $\left(\begin{array}{l}n \\ k\end{array}\right)^{\left(\begin{array}{c}2 k-1 \\ k-1\end{array}\right)}$ for the number of maximal intersecting families in $\left(\begin{array}{c}{[n]} \\ k\end{array}\right)$ obtained in [1] (see Lemma 10 for the proof of a similar statement), combined with the 
bound (13) on the size of any maximal family with such diversity, we get that

$$
\frac{I\left(n, k, \geq\left(\begin{array}{c}
n-3 \\
k-2
\end{array}\right)\right)}{2^{\left(\begin{array}{c}
n-1 \\
k-1
\end{array}\right)-\left(\begin{array}{c}
n-k-1 \\
k-1
\end{array}\right)}} \leq\left(\begin{array}{c}
n \\
k
\end{array}\right) \frac{\left(\begin{array}{c}
2 k-1 \\
k-1
\end{array}\right)}{2^{\left(\begin{array}{c}
n-1 \\
k-1
\end{array}\right)+\left(\begin{array}{c}
n-4 \\
k-3
\end{array}\right)-\left(\begin{array}{c}
n-4 \\
k-1
\end{array}\right)}} \leq 2^{n\left(\begin{array}{c}
2 k-1 \\
k-1
\end{array}\right)} 2^{\left(\begin{array}{c}
n-4 \\
k-1
\end{array}\right)-\left(\begin{array}{c}
n-k-1 \\
k-1
\end{array}\right)-\left(\begin{array}{c}
n-5 \\
k-2
\end{array}\right)}
$$

Putting $n=2 k+x$, we have $\left(\begin{array}{l}n-4 \\ k-3\end{array}\right) /\left(\begin{array}{l}n-5 \\ k-2\end{array}\right)=\frac{(n-4)(k-2)}{(n-k-1)(n-k-2)} \leq \frac{(2 k+x) k}{(k+x-2)^{2}} \leq 1-\frac{x^{2}}{(k+x)^{2}} \leq 1-\frac{1}{k}$. On the other hand,

$$
\frac{\left(\begin{array}{c}
2 k-1 \\
k-1
\end{array}\right)}{\left(\begin{array}{l}
n-5 \\
k-2
\end{array}\right)}=\frac{n-4}{k-1} \prod_{i=1}^{k} \frac{2 k-i}{n-3-i} \leq n\left(\frac{2 k}{n-3}\right)^{k} \leq \frac{1}{2 k n}
$$

where the last inequality is clearly valid for $n \geq 2 k+2+2 \sqrt{k \log k}$ and sufficiently large $k$. We conclude that the right-hand side of (15) is at most $2^{\frac{1}{2 k}\left(\begin{array}{c}n-5 \\ k-2\end{array}\right)}=o(1)$.

\section{References}

[1] J. Balogh, SA. Das, M. Delcourt, H. Liu, M. Sharifzadeh, Intersecting families of dicrete structures are typically trivial, J. Combinatorial Theory Ser. A 132 (2015), 224-245.

[2] B. Bollobás, On generalized graphs, Acta Math. Acad. Sci. Hungar. 16 (1965), 447-452.

[3] P. Erdős, C. Ko, R. Rado, Intersection theorems for systems of finite sets, The Quarterly Journal of Mathematics, 12 (1961) N1, 313-320.

[4] P. Frankl, Erdos-Ko-Rado theorem with conditions on the maximal degree, Journal of Combinatorial Theory, Series A 46 (1987), N2, 252-263.

[5] P. Frankl, N. Tokushige, Some best possible inequalities concerning cross-intersecting families, Journal of Combinatorial Theory, Series A 61 (1992), N1, 87-97.

[6] A.J.W. Hilton, The Erdös-Ko-Rado theorem with valency conditions, (1976), unpublished manuscript.

[7] A.J.W. Hilton, E.C. Milner, Some intersection theorems for systems of finite sets, Quart. J. Math. Oxford 18 (1967), 369-384.

[8] F. Jaeger, C. Payan, Nombre maximal d'aretes d'un hypergraphe critique de rang h, CR Acad. Sci. Paris 273 (1971), 221-223.

[9] G.O.H. Katona, A theorem of finite sets, "Theory of Graphs, Proc. Coll. Tihany, 1966", Akad, Kiado, Budapest, 1968; Classic Papers in Combinatorics (1987), 381-401.

[10] G.O.H. Katona, Solution of a problem of A. Ehrenfeucht and J. Mycielski, Journal of Combinatorial Theory, Series A 17 (1974), N2, 265-266. 
[11] J.B. Kruskal, The Number of Simplices in a Complex, Mathematical optimization techniques 251 (1963), 251-278.

[12] A. Kupavskii, D. Zakharov, Regular bipartite graphs and intersecting families, arXiv:1611.03129

[13] L. Lovász, Combinatorial Problems and Exercises, 13.31, North-Holland, Amsterdam (1979). 